\title{
Predictors of mortality in patients with abdominal aortic aneurysm
}

\author{
Tamer Turk ${ }^{\circledR}$, Muhammed Savran² ${ }^{(0)}$, Mesut Engin ${ }^{1 *}$
}

\section{Dear Editor;}

We have read with great interest the article by Aksoy and Uysal ${ }^{1}$ entitled "A simple risk scoring systems to evaluate the presence of aneurysm and one-year mortality in patients with abdominal aortic aneurysm using CHA2DS2-VASc and ATRIA". First of all, we congratulate the authors for their invaluable contribution to the literature. However, we would like to add some very important factors affecting mortality in patients with abdominal aortic aneurysm (AAA).

In their article, the authors aimed to investigate the effect of two scoring systems on the diagnosis of AAA and mortality in patients diagnosed with AAA. A total of 120 patients were included in the study. Firstly, patients were divided into two groups as those with AAA $(n=60)$ and those without AAA $(n=60)$, and then mortality analysis was performed on patients diagnosed with AAA. Mortality was observed in 20 (33.3\%) patients diagnosed with AAA as a result of one-year follow-up. In the multivariate analysis, in addition to a scoring system that was the subject of the study, and high blood glucose levels were determined as an independent predictors of mortality ${ }^{1}$. However, we could not obtain clear data on whether surgical or endovascular treatment was applied to patients with AAA. In the method part, we determined an exclusion criterion such as "need for preoperative resuscitation". Have surgical or endovascular procedures been applied to patients with diagnosis of $\mathrm{AAA}$ ? If they were operated, how many patients have you performed endovascular procedures?

We agree with the authors about the usability of these scoring systems in diagnosing AAA. Studies have shown that they play a role in the prognosis of cardiovascular diseases ${ }^{2}$. However, we think that the case of whether surgical or endovascular procedures were applied to the patients should be added to the multivariate analysis when performing the mortality analysis. Otherwise, the data obtained may be misleading.

In a recent study involving a large number of patients (38,008 patients), in-hospital mortality was found to be $1.07 \%$ in patients who underwent elective endovascular procedures. Also in this study, the overall survival rates were $96.2 \%$ at 6 months, $93.5 \%$ at 1 year, $88.3 \%$ at 2 years, $82.8 \%$ at 3 years, $76.2 \%$ at 4 years, $69.4 \%$ at 5 years, $63.7 \%$ at 6 years, $54.4 \%$ at 7 years, and $38.8 \%$ at 8 years. In addition, approximately $70 \%$ of the patients included in the study had an AAA diameter of $50 \mathrm{~mm}$ and more ${ }^{3}$. In the study of Aksoy and Uysal, AAA diameters were given as $53.8 \pm 7.5 \mathrm{~mm}$ versus $53.2 \pm 6.8$ in patients with a diagnosis of AAA with and without mortality, respectively, and the mortality rate was found to be $33.3 \%$ in one-year follow-up ${ }^{1}$. In a meta-analysis including 15,475 patients, the annual rupture rate was found to be a maximum of $8.2 \%$ in AAA patients with a diameter of $3-5.4 \mathrm{~cm}^{4}$.

As a result, it would be useful to discuss whether any intervention was applied while revealing the predictors of oneyear mortality in patients with a diagnosis of infrarenal AAA. Knowing the causes of death in patients with a diagnosis of AAA who did not undergo any intervention would be useful in terms of revealing the effects of the risk factors investigated in the article.

\section{AUTHORS" CONTRIBUTIONS}

TT: Conceptualization, Methodology, Writing - original draft, Writing - review \& editing. MS: Conceptualization, Methodology, Writing - original draft, Writing - review \& editing. ME: Conceptualization, Methodology, Writing - original draft, Writing - review \& editing.

\footnotetext{
'University of Health Sciences, Bursa Yuksek Ihtisas Training and Research Hospital, Department of Cardiovasculer Surgery - Bursa, Turkey.

${ }^{2}$ Agrı State Hospital, Department of Cardiovasculer Surgery - Agrı, Turkey.

*Corresponding author: mesut_kvc_cor@hotmail.com

Conflicts of interest: the authors declare there is no conflicts of interest. Funding: none.

Received on June 18, 2021. Accepted on June 27, 2021.
} 


\section{REFERENCES}

1. Aksoy F, Uysal D. A Simple risk scoring systems to evaluate the presence of aneurysm and one-year mortality in patients with abdominal aortic aneurysm using CHA2DS2-VASC and ATRIA. Rev Assoc Med Bras (1992). 2021;67(1):101-6. https:// doi.org/10.1590/1806-9282.67.01.20200487

2. Kavsur R, Becher MU, Nassan W, Sedaghat A, Aksoy A, Schrickel JW, et al. CHA2DS2-VASC score predicts coronary artery disease progression and mortality after ventricular arrhythmia in patients with implantable cardioverter-defibrillator. Int J Cardiol Heart Vasc. 2021;34:100802. https://doi.org/10.1016/j.ijcha.2021.100802
3. Hoshina K, Ishimaru S, Sasabuchi Y, Yasunaga H, Komori K, Japan Committee for Stentgraft Management (JACSM). Outcomes of endovascular repair for abdominal aortic aneurysms: a nationwide survey in Japan. Ann Surg. 2019;269(3):564-73. https://doi.org/10.1097/SLA.0000000000002508

4. Sweeting MJ, Thompson SG, Brown LC, Powell JT, RESCAN collaborators. Meta-analysis of individual patient data to examine factors affecting growth and rupture of small abdominal aortic aneurysms. Br J Surg. 2012;99(5):655-65. https://doi.org/10.1002/bjs.8707 